\title{
Effects of Aging on Efficiency of Task Switching in a Variant of the Trail Making Test
}

\author{
Timothy A. Salthouse, Jeffrey Toth, Karen Daniels, Colleen Parks, Richard Pak, \\ Michelle Wolbrette, and Kellie J. Hocking \\ Georgia Institute of Technology
}

\begin{abstract}
The Trail Making Test (TMT; R. M. Reitan, 1958, 1992) is extensively used in research in neuropsychology and in aging, in part because it has been postulated to reflect executive processes, such as planning and switching. However, neurocognitive and individual-differencebased analyses of this test are complicated because of different spatial arrangements of targets, the use of letters only in Version B, and potential order effects when Version A is administered prior to Version B. The present article examines a variant of a TMT (called the Connections Test) that attempts to remedy these deficiencies. A structural equation model suggested that there were no direct effects of age on either the nonalternating or alternating versions of the Connections Test (analogous to TMT Versions A and B, respectively); rather, all age-related effects were mediated through effects on perceptual speed. Moreover, although the nonalternating and alternating versions were strongly related to one another, only the latter had significant independent relations with measures of higher order cognition.
\end{abstract}

Several surveys (e.g., Butler, Retzlaff, \& van der Ploeg, 1991; Sellers \& Nadler, 1992) have indicated that The Trail Making Test (TMT; Reitan, 1958, 1992) is one of the most widely used tests in neuropsychological assessment. The test consists of two sheets of paper, each with 25 circles containing numbers or letters. In Version A, only numbers are in the circles, and the task is to connect the circles in numerical sequence as rapidly as possible. In Version $B$, the circles contain numbers and letters, and the task is to connect the circles in alternating numerical and alphabetical sequence (i.e., 1-A-2-B) as rapidly as possible. Scores on the test are typically expressed in terms of time, with either the time in each version, the average time across the two versions, or the difference between the times in Versions A and $B$ serving as the primary index of performance. One or more of these measures has frequently been found to be longer among people with various types of brain damage (e.g., closed head injury, frontal lobe lesions, Alzheimer's disease) and among people of older age (see reviews in Lezak, 1995; Mitrushina, Boone, \& D'Elia, 1999; Salthouse \& Fristoe, 1995; and Spreen \& Strauss, 1998).

The scores in Version B, or the difference in scores between Versions B and A, are often of greatest interest because they have been postulated to reflect aspects of executive functioning. To illustrate, Spreen and Strauss (1998) claimed that performance in Version B requires "the

Timothy A. Salthouse, Jeffrey Toth, Karen Daniels, Colleen Parks, Richard Pak, Michelle Wolbrette, and Kellie J. Hocking, School of Psychology, Georgia Institute of Technology.

This research was supported by National Institute on Aging Grant AG R3706826.

Correspondence concerning this article should be addressed to Timothy A. Salthouse, School of Psychology, Georgia Institute of Technology, Atlanta, Georgia 30332-0170. Electronic mail may be sent to tim.salthouse@psych.gatech.edu. ability to shift course during an ongoing activity" (p. 538), and Mitrushina et al. (1999) characterized Version B as assessing the "ability to alternate between sets of stimuli, an executive function" (p. 33). Although there is a clear need for measures that assess executive functioning, there are a number of limitations of the standard TMT that need to be considered before accepting it as an indicator of executive functioning. For example, Versions A and B differ in many respects in addition to the requirement to alternate between types of targets. In particular, Version A is always administered first, letters are used as targets in only Version B, only one specific search order (numbers to letters) is tested in Version B, the spatial arrangement of targets differs across versions (e.g., Arnett \& Labovitz, 1995; Fossum, Holmberg, \& Reinvang, 1992), and the distance between targets is larger in Version B than in Version A (Gaudino, Geisler, \& Squires, 1995). The extent to which these characteristics contribute to the slower performance usually found in Version B relative to Version A has not yet been fully determined, and, consequently, these confounds make interpretation of performance in Version B somewhat ambiguous. Moreover, the standard version of the TMT has a relatively large motoric requirement because of the distributed arrangement of targets, and this characteristic is not necessarily desirable because such movements may complicate evaluation of the cognitive aspects involved in the test.

This article focuses on a variant of a trail-making-type test that is based on the Zahlen Verbindsungs Test, which was introduced by Oswald and Roth (1978) and has been described in Vernon (1993). Our version of the test, which we call the Connections Test, consists of a $7 \times 7$ array of circles, with targets in four conditions consisting of numbers, letters, alternating numbers and letters, or alternating letters and numbers (see Figure 1). Successive targets are always in one of eight adjacent circles located above, below, to the left, to the right, or in one of the four diagonals 
adjacent to the target. As in the standard TMT, the task for the participant is to draw lines to connect the circles in the specified sequence as rapidly as possible. Because successive targets are always contiguous to one another and thus can be connected with simple short lines, the motoric component of our Connections Test is likely reduced relative to the standard TMT. ${ }^{1}$

One way to conceptualize the differences between the simple (nonalternating) and complex (alternating) conditions in the Connections Test is in terms of an elementary process model. For example, the following four processes can be postulated to be involved in the simple numbers or letters conditions: target identification, search-comparison, response, and sequence update. The first process corresponds to identifying the item in the sequence that serves as the target for the next response, and the last process may be analogous to incrementing an internal pointer to the next position in the relevant sequence. For some individuals, the incrementing operation with letters may be somewhat less fluent than that with numbers, and this possibility can be examined by comparing performance in the numbers-only condition with that in the letters-only condition. The searchcomparison process, in which the target is located and compared with its internal representation, and the response process, in which a line drawn from the old target to the new target, might be hypothesized to be more efficient in the Connections Test than in the standard TMT because successive targets in the Connections Test are always in one of the eight positions immediately adjacent to the prior target.

A process model for the complex condition in which the targets successively alternate between letters and numbers may include the same processes as in the simple condition, plus at least one additional process. This new process can be hypothesized to consist of switching between sequences because successive targets alternate between alphabetic and numeric sequences in these versions of the test. Other processes, such as keeping the sequences distinct and maintaining the correct position in a second sequence, may also be involved in the alternating condition, but for the present purposes, they can be grouped together with what we are calling the switching process.

We realize that this particular conceptualization of the processes involved in the simple (nonalternating) and complex (alternating) versions of trail-making-type tests is at best a crude approximation. Nevertheless, it seems clear that a major difference between the nonalternating and alternating conditions is the presence of a sequence switching component in the latter but not the former, and, thus, comparisons of performance in the simple and complex conditions of the Connections Test can be expected to be informative about processes related to sequence switching.

Results from two studies are described in this article. The purpose of the first study was to confirm the differences between simple (nonalternating sequences) and complex (alternating sequences) conditions and to examine the equivalence of several alternate forms (composed of different sequential arrangements of targets). The purpose of the second study was to investigate the relations of the scores in each condition with age and with other cognitive variables.

\section{Study 1}

\section{Method}

Participants. A total of 35 college students participated in a single session lasting about $30 \mathrm{~min}$. Unfortunately, because of a miscommunication with the research assistant, no information was obtained about the age or sex of these participants.

Procedure. Figure 1 contains an illustration of stimuli in the four conditions. The targets consisted of the numbers from 1 to 49 in the numbers condition. In the letters condition, the targets were the letters from $A$ to $Z$ followed by the letters from $A$ to $W$, with an asterisk preceding the second instance of each letter to distinguish it from the first instance. ${ }^{2}$ Targets in the numbers-letters condition consisted of the numbers from 1 to 25 alternating with the letters from $A$ to $X$, and targets in the letters-numbers condition consisted of the letters from $A$ to $\mathrm{Y}$ alternating with the numbers from 1 to 24 .

We created four different forms in each of the four conditions. The forms differed in the arrangements of successive targets, but a given form consisted of exactly the same sequence of movement directions in each of the four conditions. In all cases, the task was to draw lines to connect the targets in sequence as rapidly as possible. Because the test was administered in groups, the participants were allowed $20 \mathrm{~s}$ to work on each page, and the primary score was the number of correct connections, or lines, drawn between sequential targets.

An instruction page with samples of the numbers and numbersletters conditions was presented first to ensure that the participants understood the instructions. All participants then received the test pages in the same order: N1, L2, NL3, LN4, LN1, NL2, L3, N4, $\mathrm{N} 2, \mathrm{~L} 1, \mathrm{NL} 4, \mathrm{LN} 3, \mathrm{LN} 2, \mathrm{NL} 1, \mathrm{~L} 4, \mathrm{~N} 3$, where the letters refer to the conditions (i.e., $\mathrm{N}=$ numbers, $\mathrm{L}=$ letters, $\mathrm{NL}=$ numbers-letters, and $\mathrm{LN}=$ letters-numbers) and the digits refer to the form.

\section{Results and Discussion}

The mean numbers of correct connections and of errors (combined omissions and commissions) in the four forms and four conditions are presented in Table 1 . We conducted separate repeated measures Condition (four) $\times$ Form (four) analyses of variance on the number correct and error variables. The analysis on the number of correct connections completed in $20 \mathrm{~s}$ revealed significant $(p<.01)$ main effects of condition, $F(3,102)=349.69, M S E=31.96$, and form, $F(3,102)=5.46, M S E=17.76$, and a significant interaction of condition and form, $F(9,306)=10.04, M S E=$ 15.73. Post hoc tests revealed that the condition differences were as expected, with fewer connections in the alternating conditions (numbers-letters, letters-numbers) than in the nonalternating conditions (numbers, letters). As in the study by Salthouse and Fristoe (1995), performance in the letters

\footnotetext{
${ }^{1}$ It is important to note that contiguous targets may also decrease the sequence planning aspects of the Connections Test, relative to the standard TMT. However, as argued in the text, this change does not eliminate what is considered to be the main difference between TMT Versions A and B, namely, the need to switch or alternate between successive types of targets.

${ }^{2}$ Although this additional symbol has the potential to introduce confusion, some basis for distinguishing the first and second instances of the letters was necessary, and none of the participants reported difficulties following the sequence after this convention was explained to them.
} 
Numbers

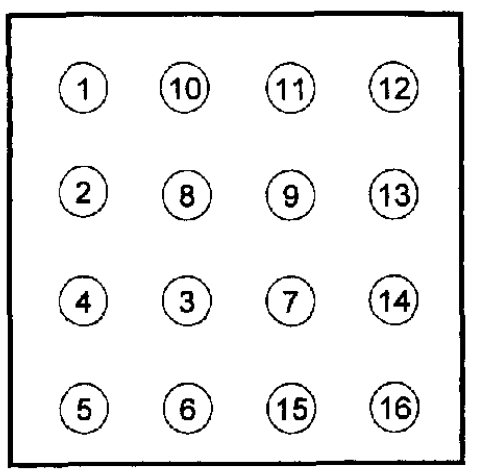

Numbers-Letters

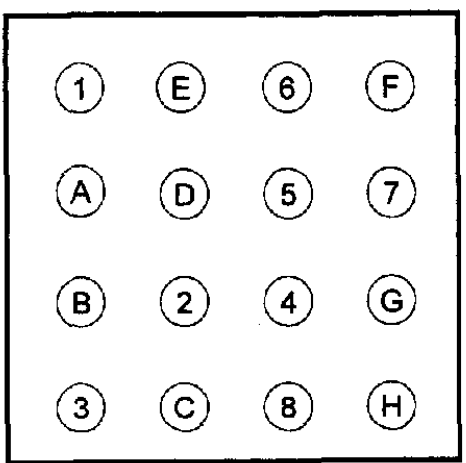

Letters

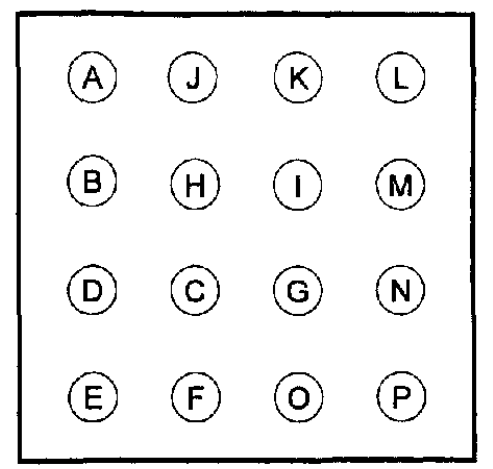

Letters-Numbers

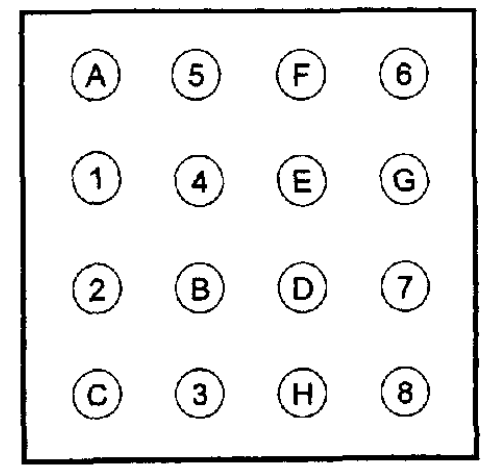

Figure 1. Illustration of arrangements of the stimuli in the four conditions of the Connections Test. It is important to note that the actual test contained 49 target circles in a $7 \times 7$ array.

condition was somewhat slower than performance in the numbers condition. There were slightly more connections completed in Form 4 than in the other forms, which may be related to the greater practice with this form because of the order in which the forms were presented. The interaction of condition and form appears to be primarily because of slower performance in the first two trials, which were in the numbers (Form 1) and letters (Form 2) conditions.

We conducted a similar analysis on the error data, but only the main effect of condition, $F(3,102)=5.49, M S E=2.31$,

Table 1

Means and Standard Deviations for the Four Conditions and the Four Forms in Study 1

\begin{tabular}{|c|c|c|c|c|c|c|c|c|c|}
\hline \multirow[b]{3}{*}{ Condition } & & \multicolumn{7}{|c|}{ Form } & \multirow[b]{3}{*}{$M$} \\
\hline & \multicolumn{2}{|c|}{1} & \multicolumn{2}{|c|}{2} & \multicolumn{2}{|c|}{3} & \multicolumn{2}{|c|}{4} & \\
\hline & $M$ & $S D$ & $M$ & $S D$ & $M$ & $S D$ & $M$ & $S D$ & \\
\hline \multicolumn{10}{|c|}{ Number correct } \\
\hline $\begin{array}{l}\text { Numbers } \\
\text { Letters } \\
\text { Numbers-letters } \\
\text { Letters-numbers } \\
\quad M\end{array}$ & $\begin{array}{l}34.9 \\
37.5 \\
22.1 \\
19.1 \\
28.4\end{array}$ & $\begin{array}{l}7.4 \\
7.4 \\
6.0 \\
4.7\end{array}$ & $\begin{array}{l}37.7 \\
31.6 \\
22.0 \\
21.9 \\
28.3\end{array}$ & $\begin{array}{l}6.0 \\
7.0 \\
5.5 \\
6.9\end{array}$ & $\begin{array}{l}37.9 \\
35.1 \\
21.8 \\
19.6 \\
28.6\end{array}$ & $\begin{array}{l}7.1 \\
8.3 \\
5.6 \\
7.0\end{array}$ & $\begin{array}{l}38.3 \\
39.1 \\
24.4 \\
18.6 \\
30.1\end{array}$ & $\begin{array}{l}6.6 \\
6.9 \\
5.6 \\
7.1\end{array}$ & $\begin{array}{l}37.2 \\
35.8 \\
22.6 \\
19.8 \\
28.9\end{array}$ \\
\hline \multicolumn{10}{|c|}{ Number of errors } \\
\hline $\begin{array}{l}\text { Numbers } \\
\text { Letters } \\
\text { Numbers-letters } \\
\text { Letters-numbers } \\
\quad M\end{array}$ & $\begin{array}{l}0.03 \\
0.43 \\
1.00 \\
0.97 \\
0.61\end{array}$ & $\begin{array}{l}0.17 \\
0.85 \\
2.20 \\
2.06\end{array}$ & $\begin{array}{l}0.23 \\
0.89 \\
0.54 \\
0.74 \\
0.60\end{array}$ & $\begin{array}{l}0.69 \\
1.45 \\
1.65 \\
1.30\end{array}$ & $\begin{array}{l}0.51 \\
0.51 \\
0.66 \\
1.09 \\
0.69\end{array}$ & $\begin{array}{l}1.72 \\
1.20 \\
1.06 \\
1.74\end{array}$ & $\begin{array}{l}0.09 \\
0.69 \\
1.09 \\
0.71 \\
0.69\end{array}$ & $\begin{array}{l}0.28 \\
2.10 \\
2.03 \\
1.72\end{array}$ & $\begin{array}{l}0.21 \\
0.63 \\
0.88 \\
0.82 \\
0.64\end{array}$ \\
\hline
\end{tabular}


$p<.01$, was significant in this analysis (other $F \mathrm{~s}<1.0$ ). A post hoc analysis revealed that there were fewer errors in the numbers condition than in any of the other conditions, which did not differ from one another. The absolute frequency of errors was low (about 2\% of total responses), which probably reflects the fact that most errors were noticed and corrected while performing the test.

In summary, the results of this initial study suggest that the different forms were reasonably equivalent, although there were clear practice effects. Of most importance, large condition effects were evident when there was a counterbalanced presentation of the conditions, arrangement of targets, and type of targets. On the average, only $58 \%$ as many connections were completed in the alternating conditions as in the nonalternating conditions.

\section{Study 2}

Because the results of the initial study were encouraging with respect to the potential of this new test, the purpose of Study 2 was to examine the relations of age to the measures of performance in each condition and the relations between those measures and other cognitive variables. Of particular interest was the extent to which there were independent age-related influences on performance in the alternating conditions, and independent relations of the measures in the alternating and nonalternating conditions with variables reflecting higher order cognition. As noted earlier, the simple (nonalternating) and complex (alternating) conditions can be hypothesized to differ primarily with respect to the addition of processes concerned with sequence switching in the alternating conditions. If these additional processes are uniquely related to age or to aspects of higher order cognition, then one would expect to find independent relations on the measures of performance in the complex conditions after taking into consideration relations involving the measures of performance in the simple conditions.

We obtained three types of cognitive variables in addition to the measures of performance in the Connections Test. We used scores on two vocabulary tests to assess product or crystallized aspects of cognition, and scores on a test of matrix reasoning and on a test of spatial visualization were used to assess process or fluid aspects of cognition. Finally, we administered tests of perceptual speed to examine potential mediating effects of perceptual speed on the relations between age and the other variables (e.g., Salthouse, Fristoe, McGuthry, \& Hambrick, 1998; Salthouse, Fristoe, \& Rhee, 1996).

\section{Method}

Participants. A total of 207 adults between 18 and 80 years of age ( $M=44.9, S D=16.1$ ) were tested individually in a single session. Descriptive information about the research participants is summarized in Table 2.

It is apparent that most of the participants had relatively high levels of education and that amount of education was positively related to age. Several of the health variables were also significantly related to age. We therefore conducted hierarchical regression analyses to examine interactions between age and the demographic and health variables on the measures of connections performance. Results of these analyses, in terms of the proportions of variance associated with the main effect of the variable and its interaction with age, are summarized in Table 3. It is important to notice that there were significant main effects for several variables in the direction of slower performance with more education, poorer health ratings, more health limitations, and reports of cardiovascular surgery or loss of consciousness. However, none of the interactions with age were significant, indicating that there was no evidence that the age relations were moderated by these variables, and, thus, we ignored the demographic and health variables in the subsequent analyses.

Procedure. Each participant received the instruction-example page and then worked on eight test pages in the following order: L1, N2, LN3, NL4, NL1, LN2, N3, and L4. As in Study 1, we allowed participants 20 s to work on each page, and the instructions were to connect the items in the appropriate sequence as rapidly as possible.

Several other pencil-and-paper cognitive tests and two reaction time tasks were also administered to all participants. One of the pencil-and-paper tests was a locally developed vocabulary test consisting of 10 antonym and 10 synonym vocabulary items. Participants had a total of $5 \mathrm{~min}$ to select which of four alternatives provided the best antonym or synonym of the target words.

A second test was the Spatial Relations Test from the Differential

Table 2

Demographic Characteristics (Means) of Participants in Study 2

\begin{tabular}{llcccccc}
\hline & \multicolumn{9}{c}{ Age decade } & Correlation \\
\cline { 2 - 8 } \multicolumn{1}{c}{ Characteristic } & $20 \mathrm{~s}$ & $30 \mathrm{~s}$ & $40 \mathrm{~s}$ & $50 \mathrm{~s}$ & $60 \mathrm{~s}$ & $70 \mathrm{~s}$ & \begin{tabular}{c} 
with age \\
\hline Age (years)
\end{tabular} \\
\cline { 2 - 9 }$n$ & 22.7 & 34.5 & 45.4 & 54.7 & 64.6 & 74.0 & - \\
\% females & 40 & 46 & 42 & 35 & 29 & 15 & - \\
Education (years) & 78 & 65 & 64 & 80 & 66 & 67 & - \\
Self-rated health & 14.5 & 16.2 & 15.7 & 15.6 & 16.6 & 16.5 & $.22 *$ \\
Health limitations & 1.9 & 1.9 & 2.1 & 1.7 & 2.0 & 2.2 & .01 \\
Cardiovascular surgery & 1.2 & 1.2 & 1.4 & 1.5 & 1.7 & 1.8 & $.23^{*}$ \\
Blood pressure medication & 0 & .02 & .05 & .03 & .13 & .15 & .17 \\
Loss of consciousness & 0 & .07 & .14 & .29 & .35 & .38 & $.33^{*}$ \\
Neurological treatment & 0 & .07 & .12 & 0 & .04 & .08 & .04 \\
\hline
\end{tabular}

Note. Self-rated health and health limitations were ratings on a scale from 1 (excellent or none) to 5 (poor or a great deal). Responses to the other four health questions were coded 1 (yes) and 0 (no), and, thus, the means can be interpreted as proportions of individuals reporting that condition. ${ }^{*} p<.01$. 
Table 3

Proportion of Variance in Connections Variables Associated With Demographic and Health Variables

\begin{tabular}{lllcc}
\hline & \multicolumn{3}{c}{ Connections variables } \\
\cline { 2 - 5 } \multicolumn{1}{c}{ Variable } & Numbers & Letters & Numbers-letters & Letters-numbers \\
\hline Sex & .004 & .013 & .005 & .004 \\
Age $\times$ Sex & .003 & .003 & .000 & .000 \\
Education (years) & $.022^{*}$ & $.031^{*}$ & $.021^{*}$ & $.041^{*}$ \\
Age $\times$ Education & .014 & .003 & .004 & .004 \\
Self-rated health & $.036^{*}$ & .024 & $.034^{*}$ & .014 \\
Age $\times$ Self-Rated Health & .000 & .000 & .002 & .000 \\
Health limitations & $.069^{*}$ & $.086^{*}$ & $.066^{*}$ & $.068^{*}$ \\
Age $\times$ Health Limitations & .001 & .003 & .007 & .001 \\
Cardiovascular surgery & .011 & .008 & $.042^{*}$ & .003 \\
Age $\times$ Cardiovascular Surgery & .000 & .022 & .001 & .000 \\
Blood pressure medication & .009 & .023 & .017 & .015 \\
Age $\times$ Blood Pressure Medication & .001 & .001 & .000 & .004 \\
Loss of consciousness & $.038^{*}$ & .026 & .006 & .002 \\
Age $\times$ Loss of Consciousness & .014 & .000 & .009 & .003 \\
Neurological treatment & .007 & .000 & .009 & .007 \\
Age $\times$ Neurological Treatment & .005 & .007 & .000 & .001 \\
\hline$* p<.01$. & & & & \\
\hline
\end{tabular}

Aptitude Test (Bennett, Seashore, \& Wesman, 1997). In this test, the respondent has to mentally assemble an unfolded piece of paper and then determine which of four 3-dimensional structures it most closely resembles. Participants are allowed $10 \mathrm{~min}$ to attempt to solve 20 problems.

A third test consisted of the first 16 odd-numbered items from the Raven's Progressive Matrices, Advanced Set II (Raven, 1962). The 16 problems were preceded by three practice problems, which were identical to the real items in consisting of a $3 \times 3$ matrix containing geometric patterns in all but the bottom right cell and a set of eight possible completions of the matrix. The task for the participant in each problem was to select the best completion of the matrix from among the eight alternatives. Participants were allotted $10 \mathrm{~min}$ to work on the 16 test problems.

A fourth test was a paired associates test involving two sets of six word pairs each. The procedure and stimulus words were the same as that described in a recent study by Salthouse et al. (1996). Briefly, each word pair was spoken by the examiner, and immediately after the last word pair, the respondent was presented a page containing the first members of each pair and was instructed to write the second member of the pair.

We also administered two pencil-and-paper perceptual speed tests. These were the Letter Comparison and Pattern Comparison Tests that have been described in several earlier studies (e.g., Salthouse et al., 1996). The tests consisted of pages containing pairs of letter strings or patterns composed of line segments, and the task for the participant was to classify each pair as same or different as rapidly as possible by writing an " $S$ " or a " $D$ " on the line between the members of the pair. Each test contained an instruction-example page followed by two test pages. We allowed the participants $30 \mathrm{~s}$ to work on each test page.

Finally, the participants also performed two reaction time tasks involving choice decisions. Both tasks consisted of the presentation of a code table at the top of the display screen and a probe in the middle of the screen. In the digit digit reaction time (DDRT) task, the code table consisted of pairs of identical digits and thus was irrelevant, and the probe stimulus consisted of a pair of digits that were either the same or different. The participant was instructed to respond with the same key ("/") if the two digits were the same and to respond with the different key (" $Z$ ") if they were different. In the digit symbol reaction time (DSRT) task, the code table consisted of the nine digits from 1 to 9 along the top row and a different symbol below each digit in the second row. The probe stimulus in this task consisted of a digit-symbol pair, and the participant was instructed to respond with the same key (" $/$ ") when the digit and symbol matched according to the code table and to respond with the different key (" $Z$ ") when the digit and symbol did not match. Each task was preceded by an 18-trial practice block and was then performed in 90-trial blocks in the order DDRT, DSRT, DSRT, and DDRT. Both accuracy and median reaction times were recorded, but the levels of accuracy averaged more than $95 \%$, and, thus, the primary measures of performance in these tasks were median reaction times in milliseconds.

\section{Results and Discussion}

We did not conduct separate analyses of errors in the Connections Test because errors were infrequent in Study 1, and they are incorporated in the number of correct connections measure because errors are subtracted when that measure is computed. The mean number of correct connections across the two forms in each condition were 27.4 for numbers, 24.4 for letters, 14.8 for numbers-letters, and 13.9 for letters-numbers. These means were considerably smaller than those for the students in Study 1 (cf. Table 1), but the values were similar when only participants in the current study under the age of 25 were considered (i.e., 34.1 for numbers, 33.0 for letters, 19.3 for numbers-letters, and 17.7 for letters-numbers). Of greatest importance, however, is that the rank ordering of conditions was the same in each sample. Furthermore, the number of connections completed in the alternating condition was about $55 \%$ of that in the nonalternating condition, which is quite similar to the value of $58 \%$ found in Study 1.

All of the scores in the Connections Test and in the perceptual speed tests were converted to seconds per item to express speed variables in the same units in which higher values corresponded to slower performance. Figure 2 portrays the means and standard errors of these time measures 


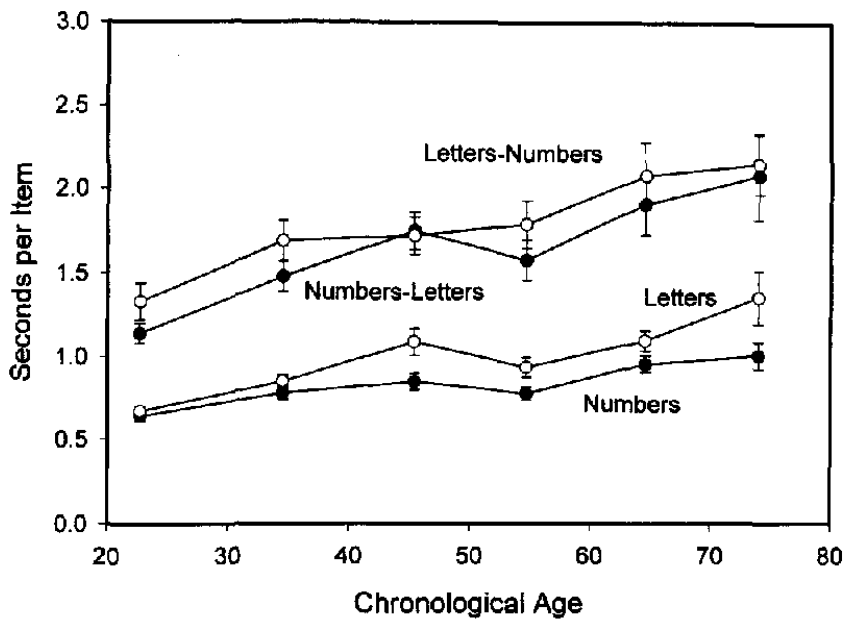

Figure 2. Means and standard errors (in seconds per item) in the four conditions of the Connections Test as a function of age decade in Study 2.

in the four connections conditions as a function of age decade. It is apparent that performance in each condition was slower with increased age, that performance in the alternating condition was slower than in the nonalternating conditions, and that performance in the letters condition was somewhat slower than that in the numbers condition. These patterns were confirmed by significant effects of age group, $F(5,201)=8.39, M S E=0.82, p<.01$; condition, $F(3$, $603)=221.28, M S E=0.18, p<.01$; and the interaction of Age $\times$ Condition, $F(15,603)=1.71, M S E=0.18, p<.01$, in addition to significant age effects in each condition (i.e., $F_{\mathrm{S}}>6.39$ ). We performed another analysis on the averages of the scores in the simple (i.e., numbers, letters) and complex (i.e., numbers-letters, letters-numbers) conditions. This analysis also revealed significant effects of age group, $F(5,201)=8.39, M S E=0.41, p<.01$; condition, $F(1$, $201)=470.35, M S E=0.12, p<.01 ;$ and an interaction of Age $\times$ Condition, $F(5,201)=2.31, M S E=0.12, p<.01$. Although the age effects were significant for both the simple (i.e., $F=10.81$ ) and complex (i.e., $F=6.21$ ) conditions, the interaction indicates that the age relations were actually somewhat larger in the simple condition than in the complex condition.

A correlation matrix with age, the connections variables, and the other cognitive variables is presented in Table 4. It is important to notice that all of the variables had respectable reliabilities, with the estimated reliabilities for the connections variables all greater than .8. Inspection of the correlation matrix reveals that most of the variables had moderate relations with one another, with the exception of generally low correlations involving the vocabulary variables. Interrelations of variables are difficult to interpret in this form, however, and, therefore, we examined the pattern among the variables in the context of a structural equation model. The two reaction time variables (DDRT and DSRT) were dropped from this analysis because data for these variables were missing from 5 participants, and the paired associates variable was dropped because it was not strongly related to the other variables used to represent higher order cognition (i.e., spatial relations and matrix reasoning; the overall patterns were similar when the analyses were repeated after

Table 4

Correlation Matrix for Variables in Study 2

\begin{tabular}{|c|c|c|c|c|c|c|c|c|c|c|c|c|c|c|}
\hline Variable & 1 & 2 & 3 & 4 & 5 & 6 & 7 & 8 & 9 & 10 & 11 & 12 & 13 & 14 \\
\hline 1. Age & - & & & & & & & & & & & & & \\
\hline 2. Letters & .41 & $(.88)$ & & & & & & & & & & & & \\
\hline 3. Numbers & .39 & .76 & $(.88)$ & & & & & & & & & & & \\
\hline 4. LetNum & .29 & .62 & .63 & $(.81)$ & & & & & & & & & & \\
\hline 5. NumLet & .34 & .64 & .68 & .59 & $(.82)$ & & & & & & & & & \\
\hline 6. LetCom & .24 & 61 & .50 & .40 & .45 & $(.75)$ & & & & & & & & \\
\hline 7. PatCom & .45 & .59 & .55 & .52 & .52 & .64 & $(.84)$ & & & & & & & \\
\hline 8. SynVoc & .41 & -.07 & -.03 & -.17 & -.09 & -.08 & -.08 & $(.83)$ & & & & & & \\
\hline 9. AntVoc & .31 & -.13 & -.10 & -.23 & -.13 & -.14 & -.17 & .83 & $(.82)$ & & & & & \\
\hline 10. SpaRel & -.31 & -.45 & -.49 & -.43 & -.46 & -.25 & -.40 & .23 & .26 & $(.87)$ & & & & \\
\hline 11. MatReas & -.42 & -.55 & -.56 & -.51 & -.53 & -.39 & -.46 & .13 & .19 & .65 & $(.77)$ & & & \\
\hline 12. PairAssoc & -.25 & -.32 & -.35 & -.39 & -.33 & -.15 & -.26 & .19 & .27 & .45 & .41 & $(.75)$ & & \\
\hline 13. DDRT & .39 & .51 & .49 & .47 & .48 & .28 & .43 & .02 & -.03 & -.40 & -.44 & -.26 & $(.70)$ & \\
\hline 14. DSRT & .55 & .69 & .62 & .57 & .57 & .43 & .58 & .06 & .01 & -.45 & -.52 & -.34 & .70 & $(.97)$ \\
\hline$M$ & 44.9 & 0.95 & 0.81 & 1.73 & 1.59 & 3.45 & 1.80 & 6.68 & 6.17 & 8.33 & 7.37 & 2.45 & 728 & 1412 \\
\hline$S D$ & 16.1 & 0.42 & 0.28 & 0.84 & 0.76 & 1.79 & 0.51 & 2.93 & 2.98 & 5.03 & 3.12 & 1.45 & 153 & 327 \\
\hline
\end{tabular}

Note. Letters, Numbers, Letters-Numbers (LetNum), and Numbers-Letters (NumLet) refer to the mean time per connection in the four conditions in the Connections Test. LetCom and PatCom refer to the time per correct comparison in the Letter Comparison and Pattern Comparison Perceptual Speed Tests (Salthouse, Fristoe, \& Rhee, 1996), and SynVoc and AntVoc refer to the number of items answered correctly in the synonym and antonym vocabulary tests. SpaRel refers to the number of items answered correctly in the Spatial Relations Test (Bennett, Seashore, \& Wesman, 1997), MatReas to the number of items answered correctly in the Raven's Progressive Matrices Test (Raven, 1962), and PairAssoc to the number of associates correctly reported in the paired associates test. DDRT and DSRT refer to median reaction time in the digit digit and digit symbol reaction time tasks. Data from the DDRT and DSRT variables based on 202 participants, but values for all other variables are based on 207 participants. Numbers in parentheses are estimated reliabilities, obtained by boosting the correlation between the scores for the two parts by the Spearman-Brown formula for Letters, Numbers, LetNum, NumLet, LetCom, PatCom, PairAssoc, DDRT, and DSRT, and from coefficient alpha for the remaining variables. Correlations with an absolute value greater than .13 were significant at $p<.05$, and those with an absolute value greater than .18 were significant at $p<.01$. 
including these variables, but the outcomes are easier to describe without these variables.)

The structural model was created by first specifying the variables assumed to represent each construct, next specifying a tentative order of the constructs in a sequence between age and the higher order cognition construct, and then determining which variables were significant in predicting each successive construct. We retained significant predictors in the model and deleted those that were not significant.

The model resulting from these steps is portrayed in Figure 3. Fit statistics for this model indicate that it provided an excellent fit to the data, $\chi^{2}(36, N=207)=59.61, p<$ .01 , nonnormed fit index $(\mathrm{NNFI})=.97$, comparative fit index $($ CFI $)=.98$, standardized root mean residual (Std. $R M R)=.04$. The chi-square test evaluates lack of fit, but it is considered too sensitive to deviations from a perfect fit to be meaningful with moderate to large samples and hence is typically supplemented by other fit statistics. The NNFI and the CFI are estimates of the degree to which the covariance matrix is reproduced more accurately by the model than by a null model in which all variables are assumed to be uncorrelated, and the Std. RMR is an estimate of the amount of unexplained or residual covariance not accounted for by the model (e.g., Kline, 1998; Maruyama, 1998). Better fits are therefore indicated by NNFI and CFI values closer to 1.0 and Std. RMR values closer to 0 .

Because the model in Figure 3 had a very high coefficient for the relation between the simple and complex connection constructs, we examined an alternative model, in which this relation was constrained to be 1.0. This constrained model fit the data significantly worse than the model in Figure $3, \chi^{2}(1$, $N=207)=60.96, p<.01$, indicating that the two constructs were distinct despite the substantial overlap of their variance.

A simpler model was also considered in which the vocabulary construct and all of its relations to other constructs were deleted. This model fit the data as well as the more complex model, $\chi^{2}(23, N=207)=41.16, p<.02$, $\mathrm{NNFI}=.97, \mathrm{CFI}=.98$, Std. RMR $=.04$, and it was similar to the model portrayed in Figure 3 in that none of the direct relations from age to either the simple or the complex connections constructs were significantly different from zero. However, the model including the vocabulary construct was preferred because vocabulary can serve as an index of crystallized intelligence that functioned to moderate some of the age-related effects on other constructs.

It is important to note that the model illustrated in Figure 3 assumes that all age-related effects on the simple condition (i.e., nonalternating targets) were mediated through effects on perceptual speed and that most of the individual differences in the complex condition (i.e., alternating targets) were mediated through differences apparent in the simple condition, with an additional influence from vocabulary. (One must remember that the paths from age to the simple and complex connection constructs were evaluated, but because they did not differ significantly from zero, they were

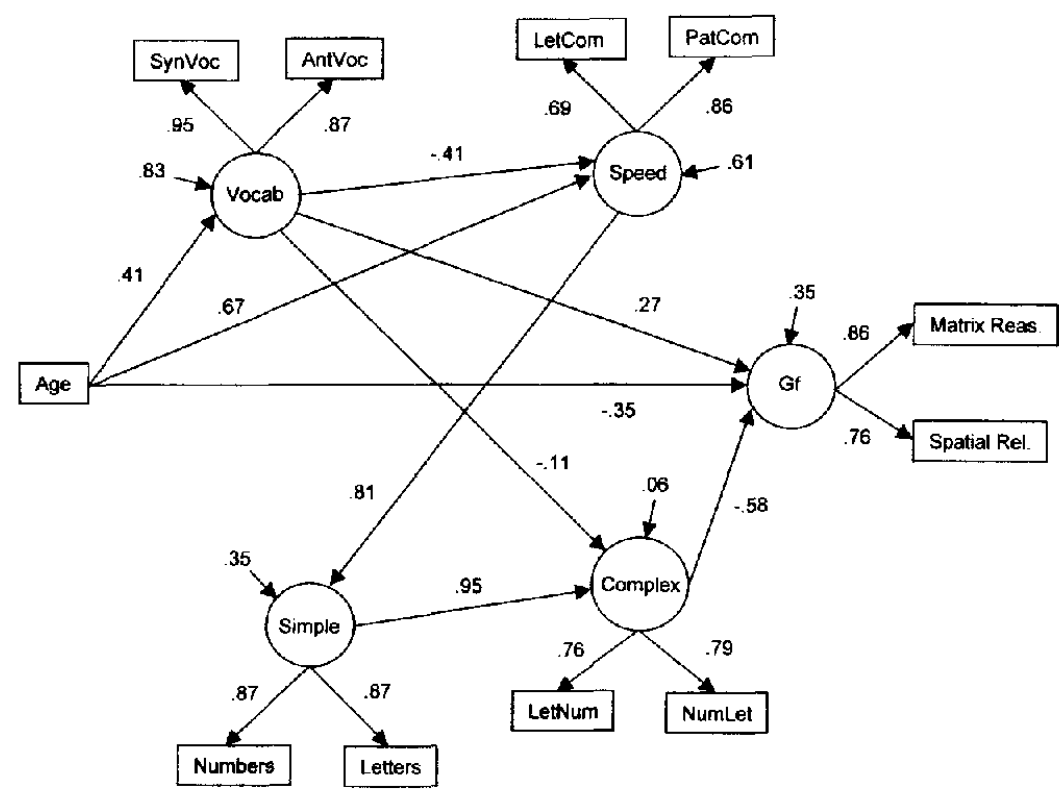

Figure 3. Structural model of interrelations among constructs in Study 2. Numbers adjacent to arrows are standardized regression coefficients, and numbers at the end of the arrows pointing to the circles correspond to the proportion of variance in the construct that was not accounted for by the predictors in the model. Simple and complex refer to the nonalternating and alternating conditions in the Connections Test, respectively, and Gf refers to fluid intelligence. SynVoc = synonym vocabulary test; AntVoc $=$ antonym vocabulary test; LetCom $=$ Letter Comparison Perceptual Speed Test (Salthouse, Fristoe, \& Rhee, 1996); PatCom = Pattern Comparison Perceptual Speed Test (Salthouse et al., 1996); Vocab = vocabulary; Reas. = reasoning; Rel. = relations; NumLet $=$ numbers-letters condition; LetNum = letters-numbers condition. 
dropped from the model.) The rationale for ordering perceptual speed before the simple and complex connections constructs was that perceptual speed can be postulated to be a component of the Connections Test because successful performance in this test seems to require an internal comparison of the generated and actual item, in addition to processes concerned with generation of the next item in a sequence, search for the target, and movement to the next item. The absence of a direct relation between perceptual speed and the higher order cognition construct in this model is probably attributable to those effects being mediated through the connection variables. The negative coefficient for the path from vocabulary to the complex condition indicates that faster performance (i.e., shorter times) in the numbers-letters and letters-numbers versions of the Connections Test was associated with higher scores in the vocabulary tests. The relations of vocabulary to perceptual speed and to the higher order cognition or fluid intelligence construct indicate that the age-related declines in these variables were partially attenuated by the positive relations between age and the vocabulary index of crystallized intelligence. In other words, this result implies that there would have been larger age-related declines in the measures of perceptual speed and fluid intelligence in the present sample if there had not been a positive relation between age and crystallized intelligence.

The measures of performance in the nonalternating and alternating conditions were very strongly related to one another, but only performance in the complex condition had significant relations with vocabulary. Furthermore, when a relation was specified from the complex condition to the higher order cognition construct, there was no effect from the simple condition to the cognition construct, indicating that there were no unique relations between performance in the nonalternating versions of the Connections Test and higher order cognition that were not shared with performance in the alternating versions.

We also conducted several sets of hierarchical regression analyses to examine the degree to which various constructs shared age-related variance. In each case, composites were created by averaging the $z$ scores for the two relevant variables in each construct (cf. Figure 3). The first analyses consisted of predicting the simple and complex connections constructs from age and either the perceptual speed (from the letter comparison and pattern comparison tests) or the reaction time speed (from the digit digit and digit symbol reaction time tasks) composite. The $R^{2}$ associated with age was .248 for the simple (nonalternating) version of the Connections Test, but this was reduced to $.024(90 \%$ reduction) after control of the perceptual speed composite, and to .032 ( $87 \%$ reduction) after control of the reaction time composite. Similar values were apparent in the prediction of the complex (alternating) version of the Connections Test, as the $R^{2}$ associated with age was .161 , and this was reduced to .006 ( $96 \%$ reduction) after control of the perceptual speed composite and to .008 (95\% reduction) after control of the reaction time composite.

The second set of analyses involved predicting performance on the alternating version of the Connections Test from age and performance on the nonalternating version. When age was the only predictor in the equation, it was associated with an $R^{2}$ of .161 , but when age was examined after control of the variance in the simple (nonalternating) condition, it was associated with an $R^{2}$ of less than .001 , which was not significantly different from zero. The results from these two sets of hierarchical regression analyses are therefore consistent with the pattern evident in Figure 3 in that virtually all of the age-related individual differences in the two versions of the Connections Test were shared with measures of perceptual (and reaction time) speed, and there was no evidence of age-related effects on performance in the complex conditions after control for effects on the simple conditions.

\section{General Discussion}

There are two major contributions of the research reported in this article; one is practical, and the other is theoretical. With respect to the practical contribution, the results of the two studies in this report suggest that the Connections Test appears promising for neuropsychological assessment because it is very brief (less than $5 \mathrm{~min}$, with two administrations in all four conditions) and yet quite reliable. Furthermore, it has numerous advantages over the standard TMT because performance in the alternating and nonalternating conditions can be compared without a confounding of the less familiar letter sequence only in the alternating condition, with minimal motor responses, and with multiple forms in each condition to control for potentially idiosyncratic arrangements of targets.

The primary theoretical contribution of the current research concerns the interpretation of the factors responsible for individual differences in the alternating and nonalternating versions of trail-making-type tests. Although TMTs have been popular in neuropsychological assessment and in research concerned with between-group comparisons (such as normal aging), there has been little consensus with respect to the nature of the factors responsible for individual differences in the nonalternating and alternating versions of the test (e.g., see reviews in Lezak, 1995; Mitrushina et al., 1999; Spreen \& Strauss, 1998). The version of the TMT examined in these studies was successful in replicating the basic phenomenon, as there were only between $55 \%$ and $58 \%$ as many connections in the alternating versions of the test as in the nonalternating versions. Moreover, this was true when the versions were presented in a counterbalanced order, when the less familiar letter sequence was included in both versions, and when the same sequence of target locations and movement directions were required in each condition.

The correlational analyses in Study 2 suggest that aspects related to perceptual speed are responsible for much of the individual differences, and particularly the age-related individual differences, in both versions of the Connections Test. This was apparent in the results of the structural model, in which nearly $66 \%$ (i.e., $.81^{2}=.656$ ) of the total variance in the simple (nonalternating) construct was associated with the perceptual speed construct, and in the hierarchical 
regression analyses, in which statistical control of the perceptual speed composite reduced the age-related variance in both the simple and complex connections measures by more than $90 \%$. Furthermore, a similar pattern was evident when speed was represented by the reaction time composite as the age-related variance in the simple connections construct was reduced by $88 \%$ after control of the reaction time composite, and the age-related variance in the complex connections construct was reduced by $95 \%$. These results therefore suggest that a large proportion of the individual differences in both the nonalternating and alternating versions of the Connections Test likely reflect differences in how quickly simple cognitive operations can be executed.

The results of Study 2 also revealed that although performance was much slower in the alternating conditions than in the nonalternating conditions, the two conditions shared a very large proportion of variance (i.e., $.95^{2}=.903$ ), and there were no independent age-related effects on the alternating conditions after statistical control of the measures of performance in the nonalternating conditions. This latter finding is consistent with earlier findings by Salthouse and Fristoe (1995), in which measures of performance in the alternating version of a computer-administered TMT were not related to age after considering the influence of age on measures of performance in the nonalternating version. Moreover, it is noteworthy that these studies involved quite different versions of the TMT (i.e., drawing lines between contiguous circles in the current studies and making keystroke responses to move a cursor between targets in the Salthouse \& Fristoe, 1995, studies). Not all studies have found this pattern, however, and it may be limited to situations in which the alternating and nonalternating versions of the tests are presented in a counterbalanced order. For example, Salthouse et al. (1996) reported a small but significant residual age-related variance in the TMT B variable (i.e., increment in $R^{2}$ of .056) after control of the TMT A variable in the standard TMT in which Version A was administered prior to Version B.

It is important to emphasize that the absence of unique or independent age-related effects on the alternating version of the test above and beyond those evident in the nonalternating version does not mean that the two versions of the test are equivalent. Not only is performance much slower in the alternating version than in the nonalternating version, which suggests that the necessity to switch between sequences involves additional time-consuming processes, but only the measure of performance in the alternating conditions was significantly related to a measure of crystallized intelligence (i.e., zero-order correlations with vocabulary were .18 for the alternating composite and .07 for the non-alternating composite). What the current results do seem to imply, however, is that although additional processes concerned with sequence switching are required in the alternating version of the test, nearly all of the age-related influences on those processes appear to be mediated through the agerelated influences on other processes that are present in the nonalternating version of the test. Salthouse et al. (1998) recently reached a similar conclusion on the basis of results from a task-switching paradigm involving pairs of reaction time tasks. Thus, the two sets of results provide converging evidence for the suggestion that there appear to be little or no specific age-related effects on one purported component of executive function; namely, the efficiency of switching between multiple tasks or between components of a single task.

No data on the Connections Test are yet available on any patient populations, and, therefore, it is possible that evidence of specific switching deficits, independent of perceptual speed differences, might be obtained in various braindamaged groups. For example, if patients with lesions in the frontal lobe are impaired in executive processes concerned with shifting or alternating between multiple activities, then they might be expected to have greater difficulty in the alternating versions (i.e., numbers-letters, letters-numbers) than in the nonalternating versions (i.e., numbers, letters) of this test. Moreover, because the Connections Test overcomes some of the limitations of the standard TMT, it may provide more sensitive assessment of the relevant processes. However, because the results of the current studies indicate that the alternating and nonalternating versions share considerable individual differences variance, it is not sufficient merely to compare absolute levels of performance on the different versions of the test. A preferable procedure is to determine the strength of the relation between the group classification variable (i.e., control vs. patient group) and performance in the alternating version of the test after the variance in the nonalternating version of the test was statistically controlled. A discovery of significant grouprelated effects in this type of analysis would provide convincing evidence for the existence of independent, and hence specific, effects on the switching processes unique to the alternating version of the test.

\section{References}

Arnett, J. A., \& Labovitz, S. S. (1995). Effect of physical layout in performance of the Trail Making Test. Psychological Assessment, 7, 220-221.

Bennett, G. K., Seashore, H. G. T., \& Wesman, A. G. (1997). Differential Aptitude Test. (1997). San Antonio, TX: Psychological Corporation.

Butler, M., Retzlaff, H. P., \& van der Ploeg, R. (1991). Neuropsychological test usage. Professional Psychology: Theory, Research, and Practice, 22, 510-512.

Fossum, B., Holmberg, H., \& Reinvang, I. (1992). Spatial and symbolic factors in performance of the Trail Making Test. Neuropsychology, 6, 71-75.

Gaudino, E. A., Geisler, M. W., \& Squires, N. K. (1995). Construct-validity in the Trail Making Test-What makes Part B harder? Journal of Clinical and Experimental Neuropsychology, $17,529-535$.

Kline, R. B. (1998). Principles and practice of structural equation modeling. New York: Guilford Press.

Lezak, M. D. (1995). Neuropsychological assessment. New York: Oxford University Press.

Maruyama, G. M. (1998). Basics of structural equation modeling. Thousand Oaks, CA: Sage. 
Mitrushina, M. N., Boone, K. B., \& D'Elia, L. F. (1999). Handbook of normative data for neuropsychological assessment. New York: Oxford University Press.

Oswald, W. D., \& Roth, E. (1978). Der Zahlen-Verbindungs Test $(Z V T)$. Gottingen, Germany: Hogrefe.

Raven, J. C. (1962). Advanced progressive matrices, Set II. London: H. K. Lewis.

Reitan, R. M. (1958). Validity of the Trail Making Test as an indication of organic brain damage. Perceptual and Motor Skills, $8,271-276$.

Reitan, R. M. (1992). Trail Making Test: Manual for administration and scoring. Tucson, AZ: Reitan Neuropsychology Laboratory.

Salthouse, T. A., \& Fristoe, N. M. (1995). Process analysis of adult age effects on a computer-administered Trail Making Test. Neuropsychology, 9, 518-528.

Salthouse, T. A., Fristoe, N., McGuthry, K., \& Hambrick, D. Z. (1998). Relation of task switching to age, speed, and fluid intelligence. Psychology and Aging, 13, 445-461.
Salthouse, T. A., Fristoe, N., \& Rhee, S. H. (1996). How localized are age-related effects on neuropsychological measures? Neuropsychology, 10, 272-285.

Sellers, A. H., \& Nadler, J. D. (1992). A survey of current neuropsychological assessment procedures used for different age groups. Psychotherapy in Private Practice, 11, 42-57.

Spreen, O., \& Strauss, E. (1998). A compendium of neuropsychological tests: Administration, norms, and commentary. New York: Oxford University Press.

Vernon, P. A. (1993). Der Zahlen-Verbindsungs Test and other trail-making correlates of general intelligence. Personality and Individual Differences, 14, 35-40.

Received December 21, 1998

Revision received June 4, 1999

Accepted June 7, 1999

\section{American Psychological Association SUBSCRIPTION CLAIMS INFORMATION}

Today's Date:

We provide this form to assist members, institutions, and nonmember individuals with any subscription problems. With the appropriate information we can begin a resolution. If you use the services of an agent, please doNOT duplicate claims through them and directly to us. PLEASE PRINT CLEARLY AND IN INK IF POSSIBLE.

\begin{tabular}{l}
\hline PRNT FULL NAME OR KEY NAME OF INSTITUTION \\
\hline ADDRESS \\
\hline CTYY \\
\hline STATE/COUNTRY
\end{tabular}

YOUR NAME AND PHONE NUMBER

TITLE

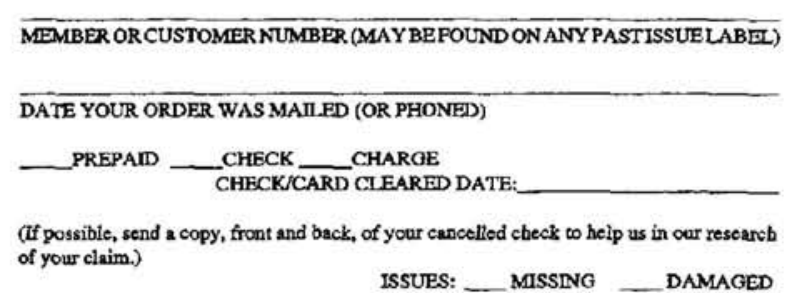

VOLUME OR YEAR
NUMBER OR MONTH

Thank you. Once a claim is received and resolved, delivery of replacement issues routinely takes 4-6 weeks.

\section{DATE RECEIVED: ACTION TAKEN: STAFF NAME:}

(TO BE FULLED OUT BY APA STAFF)

DATE OF ACTION:

INV. NO. \& DATE:

LABEL NO. \& DATE:

Send this form to APA Subscription Claims, 750 First Street, NE, Washington, DC 20002-4242

PLEASE DO NOT REMOVE. A PHOTOCOPY MAY BE USED. 